\title{
Evaluation of the pathological response and prognosis following neoadjuvant chemotherapy in molecular subtypes of breast cancer
}

This article was published in the following Dove Press journal:

OncoTargets and Therapy

19 June 2015

Number of times this article has been viewed

\section{Yue Zhao' \\ Xiaoqiu Dong ${ }^{2}$ \\ Rongguo $\mathrm{Li}^{1}$ \\ Xiao $\mathrm{Ma}^{\prime}$ \\ Jian Song' \\ Yingjie $\mathrm{Li}^{3}$ \\ Dongwei Zhang'}

'Department of General Surgery, Second Affiliated Hospital of Harbin Medical University, ${ }^{2}$ Department of Ultrasonography, Fourth Affiliated Hospital of Harbin Medical University, ${ }^{3}$ Department of Pathology, Second Affiliated Hospital of Harbin Medical University, Harbin, People's Republic of China
Correspondence: Dongwei Zhang Department of General Surgery, Second Affiliated Hospital of Harbin Medical University, 246 Xuefu Road, Nangang District, Harbin I50086, People's Republic of China

Tel +86 I350484 5465

Email mugong0@163.com
Background: The pathological complete response of neoadjuvant chemotherapy for breast cancer correlates with the prognosis for survival. Tumors may have different prognoses according to their molecular subtypes. This study was performed to evaluate the relevance of the pathological response and prognosis following neoadjuvant chemotherapy in the molecular subtypes of breast cancer.

Methods: A consecutive series of 88 patients with operable breast cancer treated with neoadjuvant chemotherapy was analyzed. Patients were classified into four molecular subtypes based on the immunohistochemistry profile of the estrogen receptor, progesterone receptor, HER2, and Ki-67. The histological response was assessed according to Miller-Payne grading (MPG) and Residual Disease in Breast and Nodes (RDBN).

Results: Ten patients (11.4\%) achieved a pathological complete response, assessed according to RDBN. The pathological complete response rate was $13.6 \%$ according to MPG. Patients with the triple-negative subtype were more likely to achieve a pathological complete response than those with luminal A breast cancer $(P=0.03)$. MPG and RDBN are independent predictors of distant disease-free survival and local recurrence-free survival, but do not predict overall survival. Ki-67, size of invasive carcinoma, lymph nodes, molecular subtypes, MPG, and RDBN are important predictors of distant disease-free survival, local recurrence-free survival, and overall survival.

Conclusion: MPG and RDBN were similarly related to the patient's prognosis. MPG was more suitable for evaluation of distant disease-free survival, and RDBN was more suitable for evaluation of local recurrence-free survival. Survival following neoadjuvant chemotherapy correlated with the pathological reaction rather than the molecular subtype of breast cancer. The molecular subtype of breast cancer was not correlated with pathological response in patients who did not achieve a pathological complete response.

Keywords: neoadjuvant chemotherapy, breast cancer, molecular subtypes, pathological response, prognosis

\section{Introduction}

Neoadjuvant chemotherapy (NACT) is often used to treat patients with locally advanced breast cancer, large tumor but operable breast cancer, or proven lymph node metastasis. The advantage of NACT is that it can downstage tumor size and increase the rate of breast-conserving surgery as well as provide information on the drug response through assessment of the changes of tumor size. ${ }^{1}$ The pathological complete response has consistently been associated with good long-term outcomes, but is achieved in only $10 \%-20 \%$ of cases. Patients who achieve a pathological complete response have a better prognosis than those who do not. ${ }^{2}$ Higher rates of pathological 
complete response can be achieved when selecting for certain breast cancer subtypes and treatment regimens. ${ }^{3}$

Breast cancer is a heterogeneous disease that can be divided into different subtypes by immunohistochemical marker expression or gene expression array data. A new surrogate intrinsic subtype was proposed at the St Gallen meeting to separate luminal A, luminal B (HER2-/HER2+), HER2-enriched, and triple-negative disease. ${ }^{4}$ Similarly, tumors may have different prognoses based on their molecular subtypes. The luminal A subtype has a better prognosis than the other subtypes, and the triple-negative subgroup has the worst prognosis. ${ }^{5,6}$ On the other hand, the triple-negative subtype is more sensitive to chemotherapy than luminal A and B breast cancer. ${ }^{7-9}$

Several histopathological classifications are available to categorize the tumor response to NACT. We chose the MillerPayne grading (MPG) and Residual Disease in Breast and Nodes (RDBN) systems to assess the pathological response of NACT. MPG provides a five-step scale based on tumor cellularity in the excision/mastectomy specimen compared with the pretreatment core biopsy as follows: grade 1, no reduction in overall cellularity; grade 2 , minor $(<30 \%)$ loss of cellularity; grade 3, estimated $30 \%-90 \%$ reduction in tumor cells; grade 4, >90\% loss of tumor cells; and grade 5, no invasive carcinoma (IC); ductal carcinoma in situ may be present. ${ }^{10}$ RDBN uses the following formula: level 1 , pathological complete response in breast and nodes, without or with carcinoma in situ, and levels $2-4$, residual disease in three different amounts, calculated as 0.2 (residual breast tumor size in $\mathrm{cm})+$ the index for the involved nodes ( 0 for no positive nodes, 1 for $1-4$ nodes, 2 for 5-7 nodes, 3 for $\geq 8$ nodes) + the Scarff-Bloom-Richardson grade (1, 2, or 3), which takes into account tumor size, lymph node stage, and histological grade to determine response. ${ }^{11-13}$

In this study, we evaluated the pathological response and prognosis following NACT in the molecular subtypes of breast cancer.

\section{Materials and methods}

\section{Patients}

The institutional review board of Harbin Medical University approved the study. We evaluated 97 patients who were initially diagnosed between 2007 and 2013 by core needle biopsy and treated with NACT followed by surgical resection at the Second Affiliated Hospital, Harbin Medical University. The pathological results showed that 88 patients had invasive ductal carcinoma, eight had invasive lobular carcinoma, and one had squamous cell carcinoma. Because invasive lobular carcinoma and squamous cell carcinoma cannot be evaluated with the RDBN system when assessing the pathological response to NACT, 13 patients were excluded. Therefore, a total of 88 patients were included in this analysis. At our institution, patients with invasive breast cancer $>3 \mathrm{~cm}$ and/ or axillary lymph nodes are typically eligible for treatment with an NACT protocol. All cases were assessed by two pathologists, and some patients without MPG were retested by two pathologists. None of the patients included in this study had metastatic disease pre-NACT. Tumor size and lymph nodes were assessed using the Seventh Edition of the American Joint Committee on Cancer staging manual pre-NACT and post-NACT. ${ }^{14}$

\section{Chemotherapy regimens and surgical treatment}

The NACT regimens comprised docetaxel, epirubicin, and cyclophosphamide (TEC; T $75 \mathrm{mg} / \mathrm{m}^{2}$ intravenously on day 1 , E $50 \mathrm{mg} / \mathrm{m}^{2}$ intravenously on day 1 , and C $500 \mathrm{mg} / \mathrm{m}^{2}$ intravenously on day 1 , repeated every 21 days); docetaxel, carboplatin, and trastuzumab/docetaxel plus trastuzumab (TCH, T $75 \mathrm{mg} / \mathrm{m}^{2}$ intravenously on day 1 , C 300/m² intravenously on day 2 , H $6 \mathrm{mg} / \mathrm{kg}$ intravenously on day 2 , repeated every 21 days $/ \mathrm{TH}$; T $75 \mathrm{mg} / \mathrm{m}^{2}$ intravenously on day 1 , H $6 \mathrm{mg} / \mathrm{kg}$ intravenously on day 1 , repeated every 21 days); and docetaxel plus cyclophosphamide (TC; T $75 \mathrm{mg} / \mathrm{m}^{2}$ intravenously on day $1, \mathrm{C} 600 \mathrm{mg} / \mathrm{m}^{2}$ intravenously on day 1 , repeated every 21 days). All patients underwent four to six cycles of NACT; five patients received $\mathrm{TCH} /$ $\mathrm{TH}$, three patients received $\mathrm{TC}$, and 80 received TEC chemotherapy regimens.

The clinical size of the primary breast cancers and axillary nodes, if the latter were palpable, was separately determined before administration of each cycle of NACT as well as before surgery. At each assessment, the product of the two greatest perpendicular diameters of the tumors in the breast and axilla was measured. Assessment of response was determined by clinical examination combined with ultrasonography. Following the NACT regimen, the response was assessed both clinically and by repeating relevant imaging. Patients with minimal or no response after two to three cycles were considered for alternative chemotherapy regimens. After the last cycle, patients were scheduled to undergo breast conservation surgery/mastectomy with axillary lymph node dissection according to patient preference, which was guided by clinical response. Patients with luminal A and B tumors underwent endocrine therapy, and positive axillary lymph nodes following NACT were treated with radiation 
therapy. Radiotherapy was postoperatively administered to patients according to standard unit protocols, and none of the patients received preoperative radiotherapy.

\section{Immunohistochemistry and molecular subtypes}

An immunohistochemical assay was used to test for expression of the estrogen receptor (ER), progesterone receptor (PR), HER2, and Ki-67. Immunohistochemistry was performed for ER (clone 1D5), PR (PgR636), HER2, and Ki-67 (clone MIB-1), all of which were from Dako (Glostrup, Denmark), as a routine diagnostic procedure. The cut-off values for ER and PR positivity were defined as $\geq 1 \%$ tumor cells with nuclear staining. Immunohistochemical staining for HER2 was scored according to standard criteria as $0,1+, 2+$, or $3+.{ }^{15}$ Scores of 0 and $1+$ were considered negative, and $3+$ was considered to be HER2-positive. When a score of 2+ was found, additional fluorescent in situ hybridization testing was performed to establish the HER2 gene amplification status. A positive result was defined as an HER2 gene/chromosome 17 ratio of $\geq 2.0$. A positive $\mathrm{Ki}-67$ finding was defined as $\geq 14 \%$, and a negative finding was defined as $<14 \%$. ${ }^{16}$ The subtype was proposed to separate the luminal A (ER+/PR+, HER2-, Ki-67 < 14\%), luminal B/HER2-(ER+/PR+, HER2-, Ki-67 $\geq 14 \%$ ), luminal B/HER2+ (ER+/PR+, HER2+), HER2-enriched (ER-, PR-, HER2+) and triple-negative (ER-, PR-, HER2-) subtypes.

\section{Statistical analysis}

Distant disease-free survival was defined as the time interval between surgery and first documented distant relapse, death, or last follow-up. Local recurrence-free survival was defined as the time interval between surgery and first documented local recurrence, death, or last follow-up. Overall survival was defined as time between surgery and death or last follow-up, whichever occurred first. Statistical analyses were performed using SAS version 9.2 software (SAS Institute, Cary, NC, USA) and GraphPad Prism version 5 (GraphPad Software Inc, La Jolla, CA, USA) was used for drawing the survival rates. The survival rates were estimated using the Kaplan-Meier product limit method. Differences between the survival curves were tested using the log rank test. The interrelated distant disease-free survival, local recurrencefree survival, and overall survival predictors were analyzed by Cox proportional hazards regression with adjustment for age by univariate and multiple regression models. Multiple Cox proportional hazard models were used to obtain the hazard ratios (HRs). Fisher's Exact test was used to assess the relationship between the different subtype groupings and pathological complete response rate. $P<0.05$ was considered to be statistically significant.

\section{Results \\ Patient characteristics, clinicopathological factors, and outcomes}

We report data from 88 patients who underwent NACT. The clinical and pathological characteristics of the study population are summarized in Table 1. All tumors were invasive ductal carcinomas without distant metastasis. The average age of the patients was 44 years. The median follow-up time was 43 months. There were seven deaths, 18 distant metastases, and 17 local recurrences. In univariate analysis, lymph nodes pre-NACT were associated with distant disease-free survival $(P=0.04)$; size of IC post-NACT $(P=0.05)$ and lymph nodes pre-NACT $(P=0.03)$ and post-NACT $(P=0.03)$ were associated with local recurrence-free survival; and size of IC pre-NACT $(P=0.03)$ and post-NACT $(P=0.03)$ was associated with overall survival.

\section{Pathological response and molecular subtype predicting survival}

MPG and RDBN classifications were used to evaluate the relevance between the pathological response and prognosis (distant disease-free survival, local recurrence-free survival, and overall survival) following NACT. In our cohort, both the MPG and RDBN systems predicted distant disease-free and local recurrence-free survival without predicting overall survival (Table 2, Figures 1 and 2). The MPG system was associated with distant disease-free survival $(P=0.04$; HR 0.623, 95\% confidence interval [CI] 0.397-0.997) and local recurrence-free survival $(P=0.02$; HR $0.579,95 \% \mathrm{CI}$ $0.36-0.93)$. The RDBN system was also associated with distant disease-free survival $(P=0.03$; HR 2.033, 95\% CI 1.058-3.909) and local recurrence-free survival $(P=0.02$; HR 2.342, 95\% CI 1.14-4.81). Neither the MPG nor RDBN was associated with overall survival in our cohort $(P=0.98$ and $P=0.70$, respectively). In our study, we compared the molecular subtype correlation with survival (Table 3 and Figure 3), and all $P$-values were $>0.05$. Therefore, molecular subtype did not correlate with distant disease-free survival, local recurrence-free survival, or overall survival.

\section{Correlation between pathological response and molecular subtype}

Tables 4 and 5 show the pathological complete response rates for each molecular subtype to determine who is more 


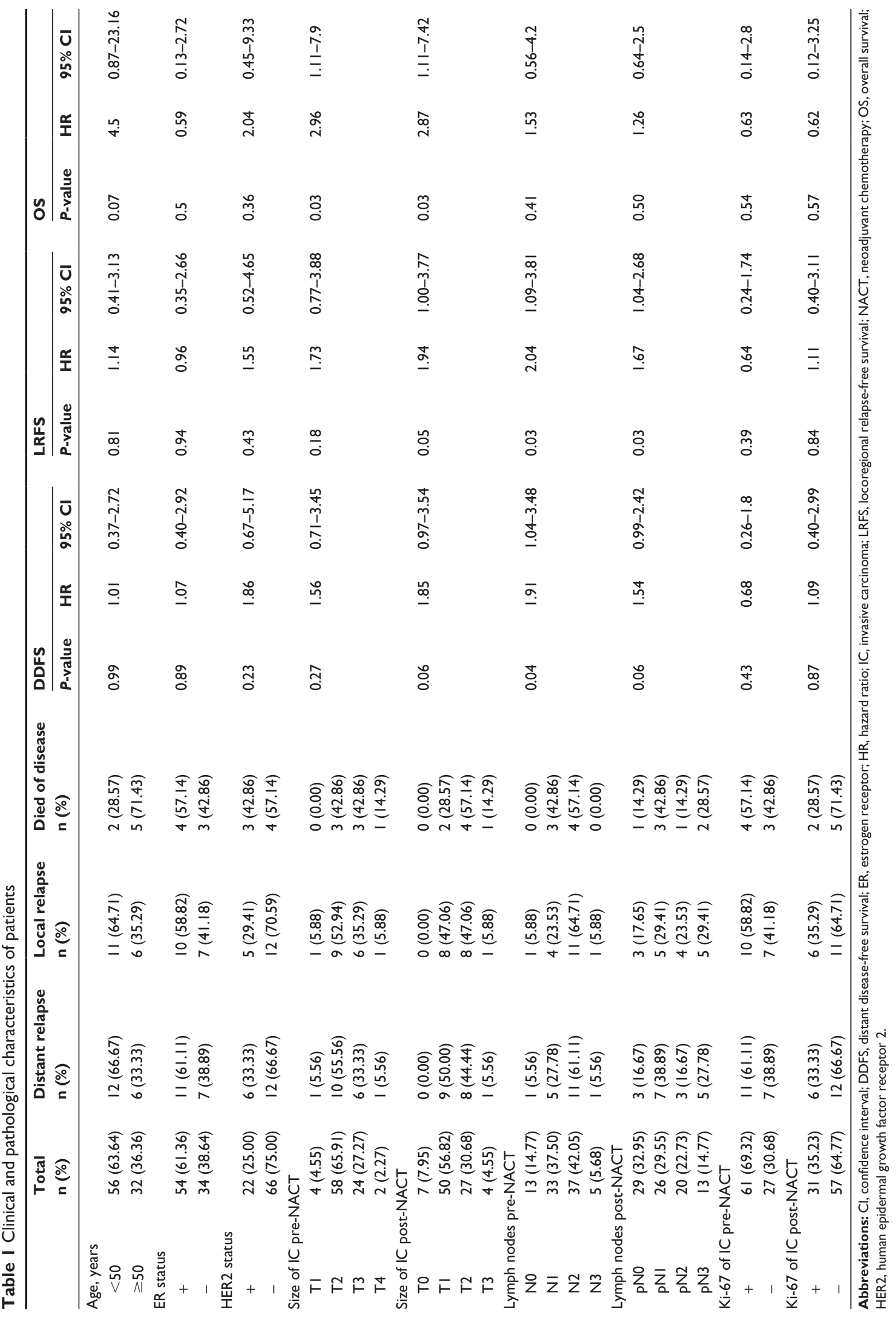




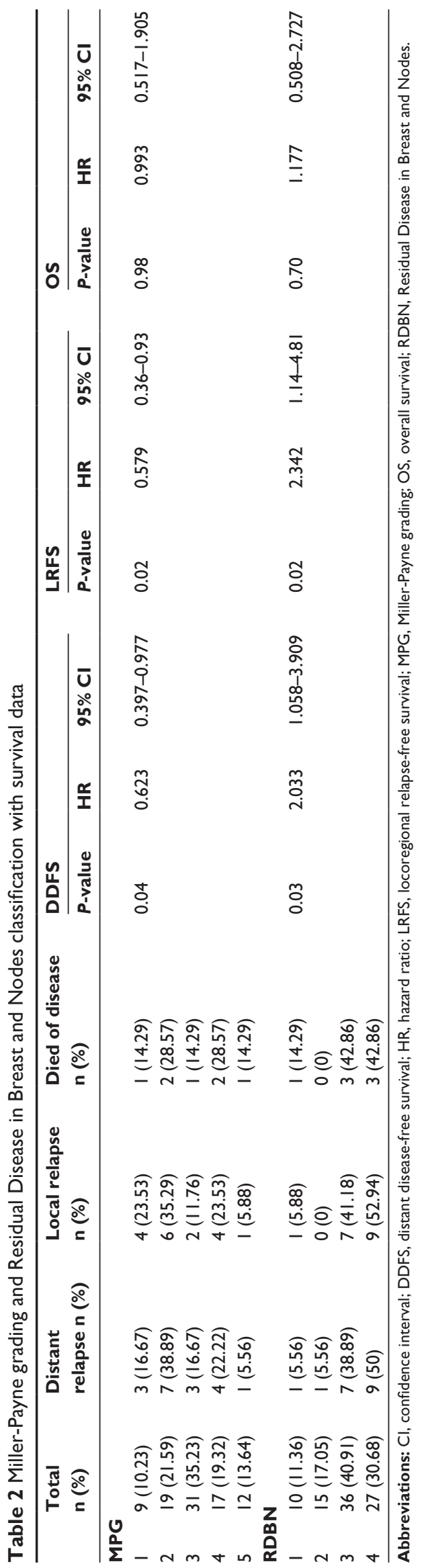

or less likely to achieve a pathological complete response after NACT. Absence of invasive tumor cells in the breast post-NACT was considered to be a pathological complete response, and was grade 5 using the MPG system. Of the 88 patients analyzed in this study, 12 achieved a tumor pathological complete response; the pathological complete response rate was $13.6 \%$. Rates of pathological complete response differed significantly between the five molecular subtypes, with $0.0 \%(0 / 31)$ for luminal A, $20.0 \%(3 / 15)$ for luminal B/HER2-, $14.3 \%$ (1/7) for luminal B/HER2+, $20.0 \%$ (3/15) for HER2-enriched, and 25.0\% (5/20) for triplenegative in Table 4 (pathological complete response 0.02 ).

The absence of invasive tumor in either breast or lymph nodes post-NACT was considered a pathological complete response, with an RDBN level of 1 . Of the 88 patients analyzed, ten achieved a tumor and lymph node pathological complete response; the pathological complete response rate was $11.4 \%$. Rates of pathological complete response differed significantly between the five molecular subtypes, with $0.0 \%(0 / 31)$ for luminal $\mathrm{A}, 13.3 \%(2 / 15)$ for luminal B/HER2-, 14.3\% (1/7) for luminal B/HER2+, 13.3\% (2/15) for HER2-enriched, and $25.0 \%$ (5/20) for triple-negative in Table 5 (pathological complete response 0.03 ).

We compared the relevance between pathological response and prognosis and between pathological response and molecular subtype. We tested the pathological complete response rate according to different molecular subtype in breast cancer patients, and all $P$-values were $<0.05$ (Tables 4 and 5). We also analyzed the MPG and RDBN classifications for molecular subtype using the Kruskal-Wallis $H$ test (Tables 6 and 7), which showed no correlation between the different molecular subtypes and the MPG and RDBN systems ( $P=0.57$ and $P=0.41$, respectively).

\section{Multivariate analysis for predicting survival}

In our study, multivariate analysis was performed to determine which factors were independent predictors of patient survival using the Cox proportional hazards regression model. Factors entered into the statistical analysis were: tumor size, lymph nodes, and Ki-67 both pre-NACT and post-NACT; ER and HER2 status; molecular subtypes; and MPG and RDBN grade.

The results of the correlative predictor analysis for distant disease-free survival, local recurrence-free survival, and overall survival are shown in Table 8 . The Ki-67 status of IC post-NACT, lymph nodes pre-NACT, MPG, and molecular subtype were interrelated predictors of distant disease-free 

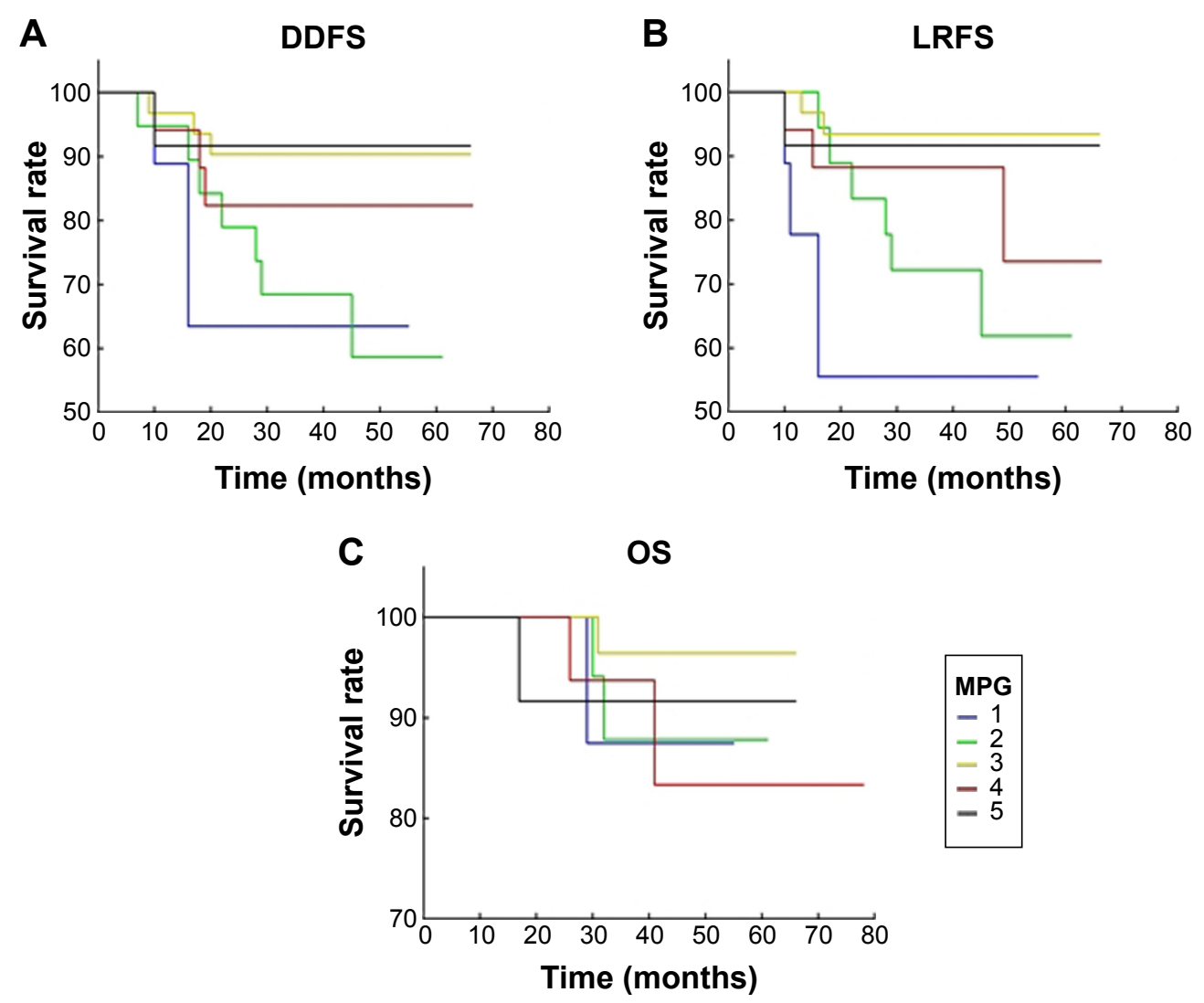

Figure I Miller-Payne grading response and survival.

Notes: (A) The Miller-Payne grading response in relation to distant disease-free survival by age-adjusted Kaplan-Meier survival analysis. The best outcome was observed in patients with pathological complete response (MPG group 5). (B) Miller-Payne grading response in relation to local recurrence-free survival by age-adjusted Kaplan-Meier survival analysis. The best outcome was observed in patients with pathological complete response (MPG group 5). (C) Miller-Payne grading response in relation to overall survival by age-adjusted Kaplan-Meier survival analysis. The best outcome was observed in the patients with pathological complete response (MPG group 5).

Abbreviations: DDFS, distant disease-free survival; LRFS, locoregional relapse-free survival; MPG, Miller-Payne grade; OS, overall survival.

survival in patients undergoing NACT (Table 8). RDBN was the only predictor of local recurrence-free survival in patients undergoing NACT (Table 8). Size of IC post-NACT, Ki-67 of IC post-NACT, and molecular subtype were predictors of overall survival in patients undergoing NACT (Table 8). Increasing MPG grade and positive status of Ki-67 of IC post-NACT were interrelated predictors of better distant disease-free survival, and lymph nodes with increasing grade pre-NACT and molecular subtype predicted worse distant disease-free survival. Increasing RDBN grade was an interrelated predictor of worse local recurrence-free survival. Positive Ki-67 status of IC post-NACT was an interrelated predictor of better overall survival, and increasing size of IC post-NACT and molecular subtype were interrelated predictors of worse overall survival (molecular subtype post-NACT 1-5 as follows: 1, luminal B/HER2+; 2, luminal A; 3, triplenegative; 4, luminal B/HER2; and 5, HER2-enriched).

\section{Discussion}

In this study, we choose the MPG and RDBN classifications to evaluate the relevance between the pathological response and prognosis (distant disease-free survival, local recurrence-free survival, overall survival) following NACT. ${ }^{17}$ MPG showed a pathological response in the breast compared with RDBN in the breast and lymph. When assessing the pathological response after NACT, both MPG and RDBN were associated with distant disease-free survival and local recurrence-free survival by age-adjusted univariate analysis (Table 2). In multivariate analysis, MPG was one of the interrelated predictors of distant disease-free survival and RDBN was the only interrelated predictor of local recurrence-free survival. However, neither MPG nor RDBN was associated with overall survival by age-adjusted univariate or multivariate analyses (Table 8). The higher the grade of MPG, the lower the distant metastasis rate and local recurrence rate, which is in contrast with results obtained using the RDBN classification method.

Tables 1 and 8 show that the MPG is the main factor of the pathological response to chemotherapy (reduction in tumor cellularity detected histologically) and can identify patients likely to have a better prognosis. A relationship between degree of histological response and 

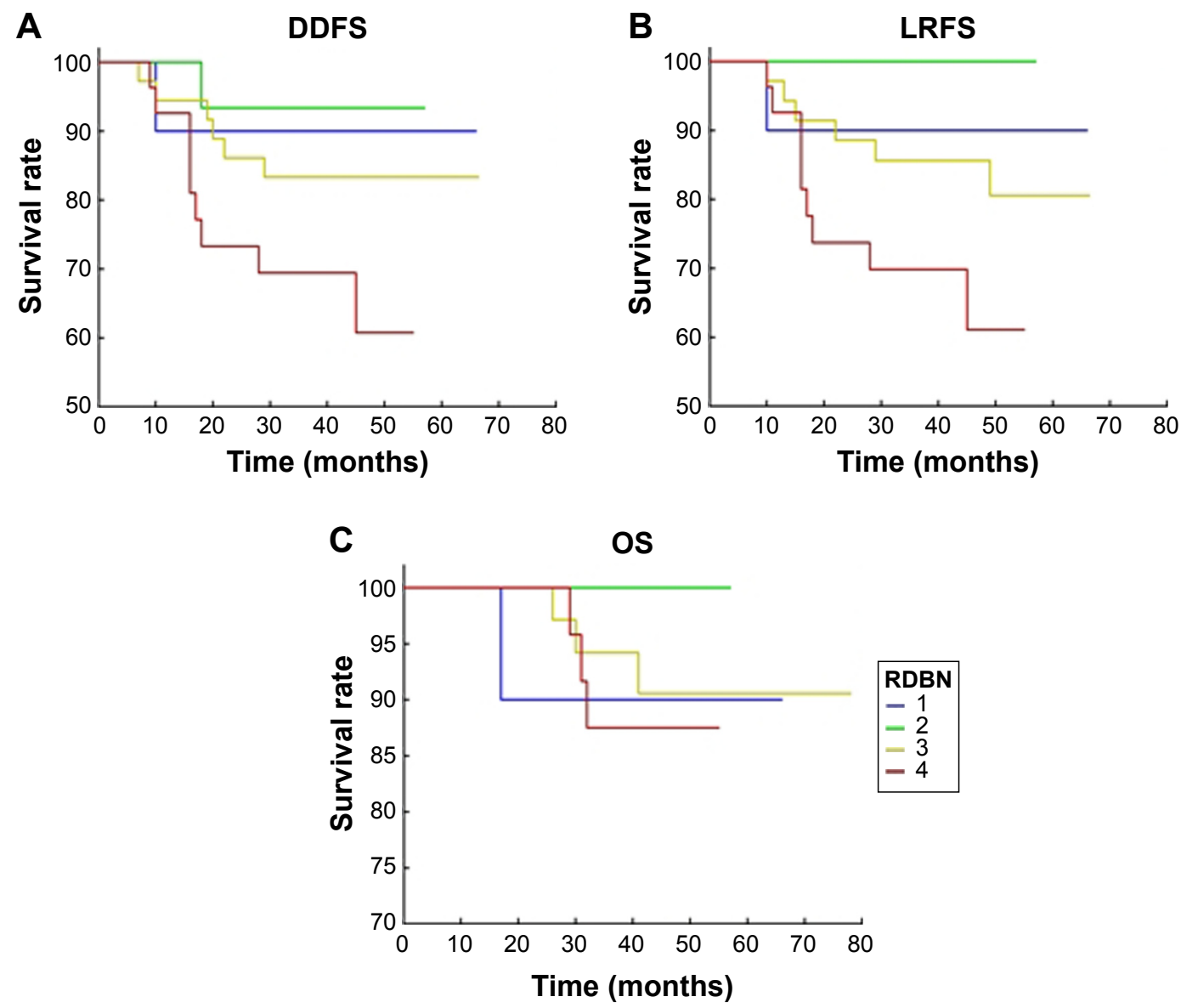

Figure 2 Residual Disease in Breast and Nodes response and survival.

Notes: (A) The Residual Disease in Breast and Nodes response in relation to distant disease-free survival by age-adjusted Kaplan-Meier survival analysis. The best outcome was observed in the patients with pathological complete response (RDBN group I). (B) The Residual Disease in Breast and Nodes response in relation to local recurrencefree survival by age-adjusted Kaplan-Meier survival analysis. The best outcome was observed in the patients with pathological complete response (RDBN group I). (C) The Residual Disease in Breast and Nodes response in relation to overall survival by age-adjusted Kaplan-Meier survival analysis. The best outcome was observed in the patients with pathological complete response (RDBN group I).

Abbreviations: DDFS, distant disease-free survival; LRFS, locoregional relapse-free survival; RDBN, Residual Disease in Breast and Nodes; OS, overall survival.

distant disease-free survival was shown in univariate and multivariate analyses $(P<0.05)$ and could potentially have an important role in the clinical management of patients with locally advanced breast cancer who are undergoing primary chemotherapy. The RDBN provided a graduation for prognostic significance and appears to be applicable after NACT. RDBN is a significant predictor of locoregional relapsefree survival in both univariate and multivariate analyses $(P<0.05)$. This classification is a useful index for evaluating the magnitude of residual disease and, subsequently, identifying subgroups of patients with a better prognosis. Lowergrade MPG was associated with more distant metastases and higher-grade RDBN was associated with more local recurrence. Therefore, MPG should be used to evaluate the distant metastasis rate and RDBN should be used to evaluate the local recurrence rate after NACT in breast cancer.

The molecular subtypes of breast cancer, which are increasingly used for predicting the pathological response to NACT, are related to histological grade and proliferative activity. ${ }^{18-20}$ Triple-negative breast cancer has the characteristics of aggressive clinical behavior, rapid growth, and a poor prognosis, but is the molecular subtype most sensitive to NACT. Luminal A tumors are considered to have high expression of hormone receptors and low expression of $\mathrm{Ki}-67$, indicating that they have a good prognosis, but they are less sensitive to chemotherapy. The relevance of molecular subtype to prognosis is shown in Table 3 (all $P>0.05$ ). The results show no correlation between the different molecular subtypes and distant disease-free survival, local recurrencefree survival, or overall survival in post-NACT univariate analysis. However, in multivariate analysis, a higher number of lymph nodes pre-NACT, and molecular subtypes indicate worse distant disease-free survival, and larger IC post-NACT and molecular subtypes indicate worse overall survival. Therefore, molecular subtype of breast cancer was not a significant predictor of survival post-NACT, and survival 


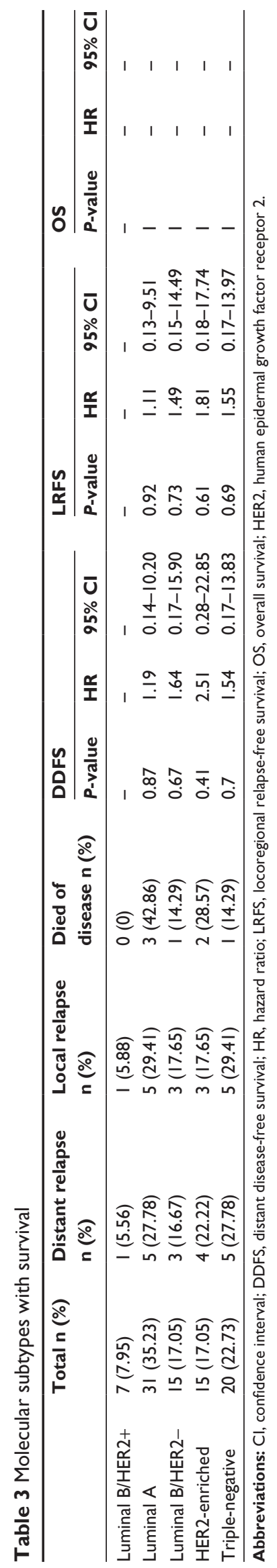

was greatly correlated with the pathological reaction in breast cancer post-NACT (Tables 2 and 3).

Many clinical trials have shown that a pathological complete response is related to good treatment outcome and could be used as a surrogate marker of better survival. ${ }^{21-23}$ Over the past 20 years, much effort has been made to identify predictive markers associated with a pathological complete response. ER and PR levels, HER-2/neu gene status, grade and subtype of IC, extensive tumor necrosis, invasion of the vascular space, and markers of proliferation have been inconsistently linked with the pathological complete response. ${ }^{24-26}$ Grade 5 in the MPG system and level 1 in the RDBN system were considered indicative of a pathological complete response (Tables 4 and 5). Patients with a pathological complete response in this study included those with no residual tumor in either the breast or lymph nodes (RDBN level $1, \mathrm{n}=10$ ) as well as those with no residual tumor in the breast alone (MPG grade 5, $\mathrm{n}=12$ ). For economic reasons, there were only five patients with trastuzumab, pathological complete response evaluation in luminal B/HER2+ and HER2-enriched subtypes, whose results were limitations. Only one patient, who was MPG grade 5 and RDBN level, developed distant metastasis and local recurrence, and died of the disease. This patient had inflammatory breast cancer, reached a pathological complete response after five cycles, but died within 17 months of NACT. In the 88 patients, pathological complete response rates for the breast alone in luminal A, luminal B/HER2-, luminal B/HER2+, HER2 overexpression, and triple-negative disease were $0.0 \%$, $20.0 \%, 14.3 \%, 20.0 \%$, and $25.0 \%(P=0.02)$, respectively. The pathological complete response rates in the breast and nodes of luminal A, luminal B/HER2-, luminal B/HER2+, HER2-enriched, and triple-negative disease were $0.0 \%$, $13.3 \%, 14.3 \%, 13.3 \%$, and $25.0 \%(P=0.03)$, respectively. We identified a subset of carcinoma patients who were most likely to achieve a pathological complete response from NACT (triple-negative, 25.0\%) and identified a group who were least likely to sustain a pathological complete response (luminal A, 0.0\%) in both the MPG and RDBN classifications. Although luminal A tumors are considered to be less responsive to chemotherapy, high hormone receptor expression and low Ki-67 expression seem to indicate a good prognosis..$^{27,28}$ Patients with triple-negative breast cancer achieved a pathological complete response and had a better prognosis than those who did not. ${ }^{29,30}$

We also compared the correlation between the molecular subtypes and pathological response using the Kruskal-Wallis $H$ test (Tables 6 and 7). Although there was no correlation 

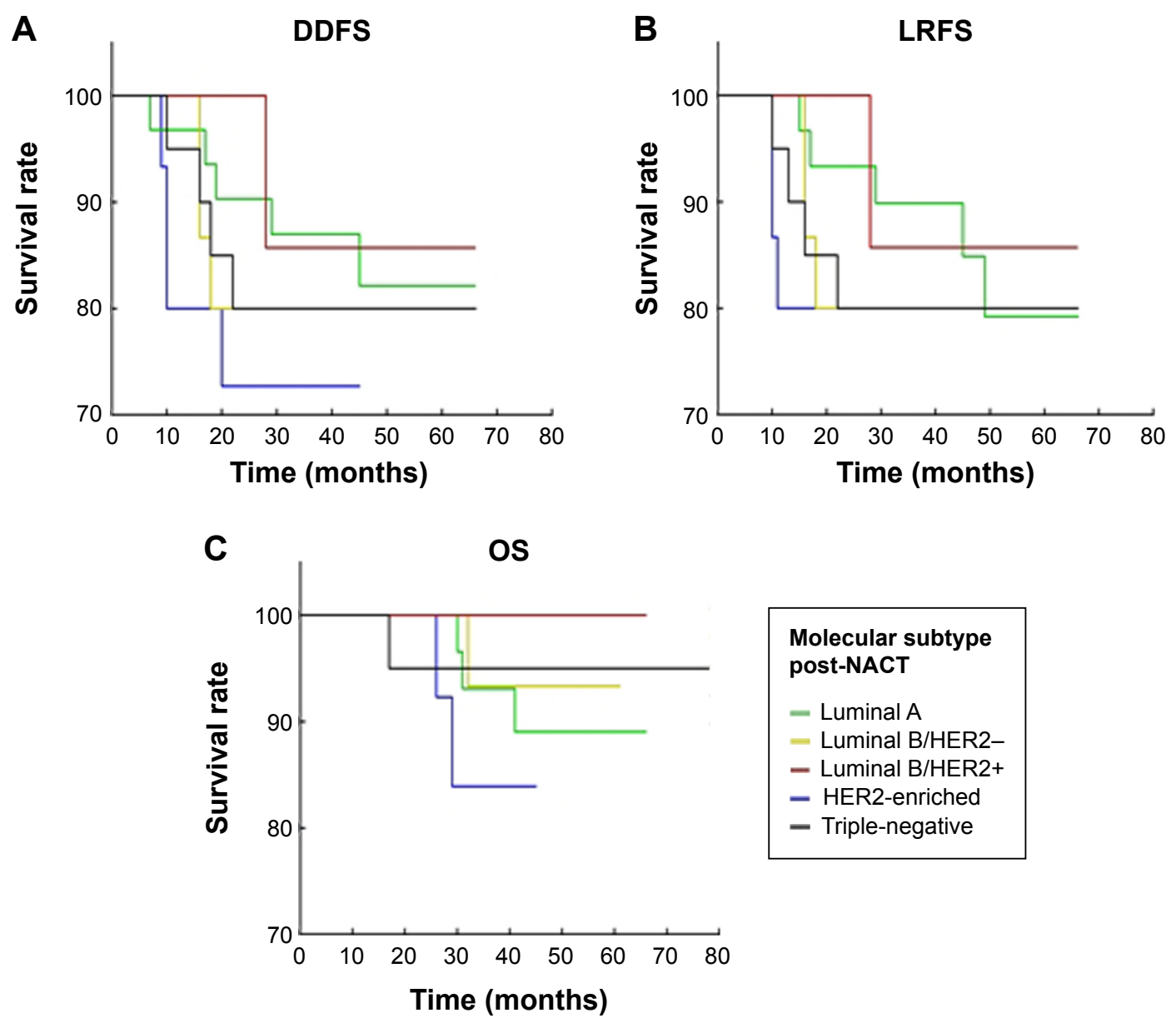

Molecular subtype post-NACT

- Luminal A

- Luminal B/HER2-

- Luminal B/HER2+

- HER2-enriched

- Triple-negative

Figure 3 Molecular subtypes and survival.

Notes: (A) Molecular subtypes in relation to distant disease-free survival by age-adjusted Kaplan-Meier survival analysis. (B) The molecular subtypes in relation to local recurrence-free survival by age-adjusted Kaplan-Meier survival analysis. (C) Molecular subtypes in relation to overall survival by age-adjusted Kaplan-Meier survival analysis.

Abbreviations: DDFS, distant disease-free survival; LRFS, locoregional relapse-free survival; NACT, neoadjuvant chemotherapy; OS, overall survival.

Table 4 Miller-Payne grade 5 (pCR) with molecular subtypes

\begin{tabular}{|c|c|c|c|c|c|c|c|}
\hline \multirow[t]{2}{*}{ Molecular subtype } & \multicolumn{2}{|c|}{ Total } & \multicolumn{2}{|c|}{ pCR } & \multicolumn{2}{|c|}{ Non-pCR } & \multirow[t]{2}{*}{$P$-value } \\
\hline & $\mathbf{n}$ & $\%$ & $\mathbf{n}$ & $\%$ & $\mathbf{n}$ & $\%$ & \\
\hline Luminal A & 31 & 35.23 & 0 & 0.00 & 31 & 40.79 & 0.02 \\
\hline Luminal B/HER2+ & 7 & 7.95 & 1 & 8.33 & 6 & 7.89 & \\
\hline Luminal B/HER2- & 15 & 17.05 & 3 & 25.00 & 12 & 15.79 & \\
\hline HER2-enriched & 15 & 17.05 & 3 & 25.00 & 12 & 15.79 & \\
\hline Triple-negative & 20 & 22.73 & 5 & 41.67 & 15 & 19.74 & \\
\hline
\end{tabular}

Abbreviations: pCR, pathological complete response; HER2, human epidermal growth factor receptor 2.

Table 5 Residual Disease in Breast and Nodes classification level I (pCR) with molecular subtypes

\begin{tabular}{|c|c|c|c|c|c|c|c|}
\hline \multirow[t]{2}{*}{ Molecular subtype } & \multicolumn{2}{|c|}{ Total } & \multicolumn{2}{|c|}{ pCR } & \multicolumn{2}{|c|}{ Non-pCR } & \multirow[t]{2}{*}{$P$-value } \\
\hline & $\mathbf{n}$ & $\%$ & $\mathbf{n}$ & $\%$ & $\mathbf{n}$ & $\%$ & \\
\hline Luminal A & 31 & 35.23 & 0 & 0.00 & 31 & 39.47 & 0.03 \\
\hline Luminal B/HER2+ & 7 & 7.95 & 1 & 10.00 & 6 & 7.69 & \\
\hline Luminal B/HER2- & 15 & 17.05 & 2 & 20.00 & 13 & 16.67 & \\
\hline HER2-enriched & 15 & 17.05 & 2 & 20.00 & 13 & 16.67 & \\
\hline Triple-negative & 20 & 22.73 & 5 & 50.00 & 15 & 19.23 & \\
\hline
\end{tabular}

Abbreviations: pCR, pathological complete response; HER2, human epidermal growth factor receptor 2. 
Table 6 Miller-Payne grade with molecular subtypes

\begin{tabular}{|c|c|c|c|c|c|c|c|c|c|c|c|c|}
\hline \multirow[t]{2}{*}{ MPG } & \multicolumn{2}{|c|}{ Luminal A } & \multicolumn{2}{|c|}{ Luminal B/HER2- } & \multicolumn{2}{|c|}{ Luminal B/HER2+ } & \multicolumn{2}{|c|}{ HER2-enriched } & \multicolumn{2}{|c|}{ Triple-negative } & \multirow[t]{2}{*}{$\chi^{2}$} & \multirow[t]{2}{*}{$P$-value } \\
\hline & n & $\%$ & $\mathbf{n}$ & $\%$ & n & $\%$ & n & $\%$ & n & $\%$ & & \\
\hline 1 & 0 & 0.00 & 4 & 26.67 & 0 & 0.00 & 2 & 13.33 & 3 & 15.00 & 2.91 & 0.57 \\
\hline 2 & 8 & 25.81 & 4 & 26.67 & $\mathrm{I}$ & 14.29 & 2 & 13.33 & 4 & 20.00 & & \\
\hline 3 & 15 & 48.39 & 3 & 20.00 & 3 & 42.86 & 5 & 33.33 & 5 & 25.00 & & \\
\hline 4 & 8 & 25.81 & I & 6.67 & 2 & 28.57 & 3 & 20.00 & 3 & 15.00 & & \\
\hline 5 & 0 & 0.00 & 3 & 20.00 & 1 & 14.29 & 3 & 20.00 & 5 & 25.00 & & \\
\hline Total & 31 & 100.00 & 15 & 100.00 & 7 & 100.00 & 15 & 100.00 & 20 & 100.00 & & \\
\hline
\end{tabular}

Abbreviations: MPG, Miller-Payne grade; HER2, human epidermal growth factor receptor 2.

Table 7 Residual Disease in Breast and Nodes classification with molecular subtypes

\begin{tabular}{|c|c|c|c|c|c|c|c|c|c|c|c|c|}
\hline \multirow[t]{2}{*}{ RDBN } & \multicolumn{2}{|c|}{ Luminal A } & \multicolumn{2}{|c|}{ Luminal B/HER2- } & \multicolumn{2}{|c|}{ Luminal B/HER2+ } & \multicolumn{2}{|c|}{ HER2-enriched } & \multicolumn{2}{|c|}{ Triple-negative } & \multirow[t]{2}{*}{$x^{2}$} & \multirow[t]{2}{*}{$P$-value } \\
\hline & $\mathbf{n}$ & $\%$ & $\mathbf{n}$ & $\%$ & $\mathbf{n}$ & $\%$ & $\mathbf{n}$ & $\%$ & $\mathbf{n}$ & $\%$ & & \\
\hline I & 0 & 0.00 & 2 & 13.33 & 1 & 14.29 & 2 & 13.33 & 5 & 25.00 & 3.99 & $0.4 I$ \\
\hline 2 & 6 & 19.35 & 1 & 6.67 & 2 & 28.57 & 3 & 20.00 & 3 & 15.00 & & \\
\hline 3 & 15 & 48.39 & 6 & 40.00 & 3 & 42.86 & 5 & 33.33 & 7 & 35.00 & & \\
\hline 4 & 10 & 32.26 & 6 & 40.00 & 1 & 14.29 & 5 & 33.33 & 5 & 25.00 & & \\
\hline Total & 31 & 100.00 & 15 & 100.00 & 7 & 100.00 & 15 & 100.00 & 20 & 100.00 & & \\
\hline
\end{tabular}

Abbreviations: RDBN, Residual Disease in Breast and Nodes; HER2, human epidermal growth factor receptor 2.

between the different molecular subtypes and MPG and RDBN classification, patients with the triple-negative disease subtype were more likely to achieve a pathological complete response (RDBN level 1/MPG grade 5) than those with the other molecular subtypes of breast cancer. As a result, the molecular subtypes of breast cancer did not correlate with pathological response for those who did not achieve a pathological complete response.

Overall survival, distant disease-free survival, and local recurrence-free survival were the outcomes of interest in the prognostic analyses. Distant relapse and local recurrence impacted the quality of survival in patients. Distant diseasefree survival, local recurrence-free survival, and overall survival were evaluated in single-factor and multiple-factor analysis. This study investigated the value of Ki-67, size of
IC, lymph nodes, molecular subtypes, MPG and RDBN as predictive factors in relation to NACT as well as the possible effects on prognosis. MPG was found to be an independent predictor for distant disease-free survival compared with RDBN for local recurrence-free survival in both univariate and multivariate analyses.

\section{Conclusion}

In conclusion, this study shows that MPG and RDBN are independent predictors of distant disease-free survival and local recurrence-free survival. However, MPG should be used to evaluate the distant metastasis rate and RDBN should be used to evaluate the local recurrence rate after NACT in breast cancer. With regard to molecular subtype,

Table 8 Distant disease-free survival, locoregional relapse-free survival, and overall survival in multivariate analysis

\begin{tabular}{|c|c|c|c|c|c|c|c|}
\hline & \multirow{2}{*}{$\begin{array}{l}\text { Parameter } \\
\text { estimate }\end{array}$} & \multirow[t]{2}{*}{ SE } & \multirow[t]{2}{*}{$\chi^{2}$} & \multirow[t]{2}{*}{$P$-value } & \multirow[t]{2}{*}{ HR } & \multicolumn{2}{|l|}{$95 \% \mathrm{Cl}$} \\
\hline & & & & & & Lower limit & Upper limit \\
\hline \multicolumn{8}{|l|}{ DDFS } \\
\hline $\mathrm{Ki}-67$ of IC post-NACT & -1.78 & 0.80 & 4.93 & 0.03 & 0.17 & 0.04 & 0.81 \\
\hline Lymph nodes pre-NACT & 0.75 & 0.34 & 4.94 & 0.03 & 2.11 & 1.09 & 4.07 \\
\hline MPG & -0.82 & 0.28 & 8.37 & $<0.01$ & 0.44 & 0.25 & 0.77 \\
\hline Molecular subtype & 0.71 & 0.28 & 6.61 & 0.01 & 2.04 & 1.19 & 3.52 \\
\hline \multicolumn{8}{|l|}{ LRFS } \\
\hline RDBN & 0.85 & 0.37 & 5.37 & 0.02 & 2.3 & 1.14 & 4.81 \\
\hline \multicolumn{8}{|l|}{ OS } \\
\hline Size of IC post-NACT & 2.07 & 0.67 & 9.44 & $<0.01$ & 7.90 & 2.11 & 29.53 \\
\hline $\mathrm{Ki}-67$ of IC post-NACT & -3.30 & 1.29 & 6.56 & 0.01 & 0.08 & 0.00 & 0.46 \\
\hline Molecular subtype & 0.87 & 0.36 & 5.84 & 0.02 & 2.39 & 1.18 & 4.83 \\
\hline
\end{tabular}

Abbreviations: IC, invasive carcinoma; $\mathrm{Cl}$, confidence interval; DDFS, distant disease-free survival; HR, hazard ratio; LRFS, locoregional relapse-free survival; NACT, neoadjuvant chemotherapy; OS, overall survival; RDBN, Residual Disease in Breast and Nodes; MPG, Miller-Payne grade; SE, standard error. 
patients with the triple-negative subtype were more likely to achieve a pathological complete response than those with other molecular subtypes of breast cancer. Survival postNACT was correlated with the pathological reaction rather than with the molecular subtype of breast cancer. Further, survival rate is improved by NACT in view of the different predictive factors.

\section{Disclosure}

The authors report no conflicts of interest in this work.

\section{References}

1. Mieog JS, van der Hage JA, van de Velde CJ. Neoadjuvant chemotherapy for operable breast cancer. Br J Surg. 2007;94:1189-1200.

2. Rastogi P, Anderson SJ, Bear HD, et al. Preoperative chemotherapy: updates of National Surgical Adjuvant Breast and Bowel Project Protocols B-18 and B-27. J Clin Oncol. 2008;26:778-785.

3. von Minckwitz G, Untch M, Blohmer JU, et al. Definition and impact of pathologic complete response on prognosis after neoadjuvant chemotherapy in various intrinsic breast cancer subtypes. J Clin Oncol. 2012;30:1796-1804.

4. Goldhirsch A, Wood WC, Coates AS, et al. Strategies for subtypes dealing with the diversity of breast cancer: highlights of the St Gallen International Expert Consensus on the Primary Therapy of Early Breast Cancer 2011. Ann Oncol. 2011;22:1736-1747.

5. Perou CM, Sorlie T, Eisen MB, et al. Molecular portraits of human breast. Nature. 2000;406:747-752.

6. Hu Z, Fan C, Oh DS, et al. The molecular portraits of breast tumors are conserved across microarray platforms. BMC Genomics. 2006;7:96.

7. Carey LA, Dees EC, Sawyer L, et al. The triple negative paradox: primary tumor chemosensitivity of breast cancer subtypes. Clin Cancer Res. 2007;13:2329-2334.

8. Goldstein NS, Decker D, Severson D, et al. Molecular classification system identifies invasive breast carcinoma patients who are most likely and those who are least likely to achieve a complete pathologic response after neoadjuvant chemotherapy. Cancer. 2007;110:1687-1696.

9. Straver ME, Glas AM, Hannemann J, et al. The 70-gene signature as a response Neoadjuvant Chemotherapy in Operable Breast Cancer 2417 predictor for neoadjuvant chemotherapy in breast cancer. Breast Cancer Res Treat. 2010;119:551-558.

10. Ogston KN, Miller ID, Payne S, et al. A new histological grading system to assess response of breast cancers to primary chemotherapy: prognostic significance and survival. Breast. 2003;12:320-327.

11. Chollet $\mathrm{P}$, Amat $\mathrm{S}$, Belembaogo E, et al. Is Nottingham prognostic index useful after induction chemotherapy in operable breast cancer? Br J Cancer. 2003;89:1185-1191.

12. Chollet $\mathrm{P}, \mathrm{Abrial} \mathrm{C}$, Durando $\mathrm{X}$, et al. A new prognostic classification after primary chemotherapy for breast cancer: residual disease in breast and nodes (RDBN). Cancer J. 2008;14:128-132.

13. Abrial SC, Penault-Llorca F, Delva R, et al. High prognostic significance of residual disease after neoadjuvant chemotherapy: a retrospective study in 710 patients with operable breast cancer. Breast Cancer Res Treat. 2005;94:255-263.

OncoTargets and Therapy

\section{Publish your work in this journal}

OncoTargets and Therapy is an international, peer-reviewed, open access journal focusing on the pathological basis of all cancers, potential targets for therapy and treatment protocols employed to improve the management of cancer patients. The journal also focuses on the impact of management programs and new therapeutic agents and protocols on
14. Edge SB, Byrd SB, Compton CC, Fritz AG, Greene FL, Trotti A, editors. American Joint Committee on Cancer (AJCC) Cancer Staging Manual. 7th ed. New York, NY, USA: Springer; 2010.

15. Sauter G, Lee J, Bartlett JM, Slamon DJ, Press MF. Guidelines for human epidermal growth factor receptor 2 testing: biologic and methodologic considerations. J Clin Oncol. 2009;27:1323-1333.

16. Cheang MC, Chia SK, Voduc D, et al. Ki67 index, HER2 status, and prognosis of patients with luminal B breast cancer. J Natl Cancer Inst. 2009; 101:736-750.

17. Straver ME, Rutgers EJ, Rodenhuis S, et al. The relevance of breast cancer subtypes in the outcome of neoadjuvant chemotherapy. Ann Surg Oncol. 2010;17:2411-2418.

18. Parker JS, Mullins M, Cheang MC, et al. Supervised risk predictor of breast cancer based on intrinsic subtypes. J Clin Oncol. 2009;27: $1160-1167$.

19. Tordai A, Wang J, Andre F, et al. Evaluation of biological pathways involved in chemotherapy response in breast cancer. Breast Cancer Res. 2008;10:R37.

20. Klauber-DeMore N, Ollila DW, Moore DT, et al. Size of residual lymph node metastasis after neoadjuvant chemotherapy in locally advanced breast cancer patients is prognostic. Ann Surg Oncol. 2006;13:685-691.

21. Romero A, Martín M, Cheang MC, et al. Assessment of topoisomerase II $\alpha$ status in breast cancer by quantitative PCR, gene expression microarrays, immunohistochemistry, and fluorescence in situ hybridization. $\mathrm{Am}$ $J$ Pathol. 2011;178:1453-1460.

22. Caudle AS, Gonzalez-Angulo AM, et al. Predictors of tumor progression during neoadjuvant chemotherapy in breast cancer. $J$ Clin Oncol. 2010;28:1821-1828.

23. Montagna E, Bagnardi V, Rotmensz N, et al. Pathological complete response after preoperative systemic therapy and outcome: relevance of clinical and biologic baseline features. Breast Cancer Res Treat. 2010; 124:689-699.

24. Penault-Llorca F, Cayre A, Bouchet MF, et al. Induction chemotherapy for breast carcinoma: predictive markers and relation with outcome. Int J Oncol. 2003;22:1319-1325.

25. Billgren AM, Rutqvist LE, Tani E, Wilking N, Fornander T, Skoog L. Proliferating fraction during neoadjuvant chemotherapy of primary breast cancer in relation to objective local response and relapse-free survival. Acta Oncol. 1999;38:597-601.

26. Davis DW, Buchholz TA, Hess KR, Sahin AA, Valero V, McConkey DJ. Automated quantification of apoptosis after neoadjuvant chemotherapy for breast cancer: early assessment predicts clinical response. Clin Cancer Res. 2003;9:955-960.

27. Penault-Llorca F, Abrial C, Raoelfils I, et al. Comparison of the prognostic significance of Chevallier and Sataloff's pathologic classifications after neoadjuvant chemotherapy of operable breast cancer. Hum Pathol. 2008;39:1221-1228.

28. Bhargava R, Beriwal S, Dabbs DJ, et al. Immunohistochemical surrogate markers of breast cancer molecular classes predicts response to neoadjuvant chemotherapy: a single institutional experience with 359 cases. Cancer. 2010;116:1431-1439.

29. Liedtke C, Mazouni C, Hess KR, et al. Response to neoadjuvant therapy and long-term survival in patients with triple-negative breast cancer. J Clin Oncol. 2008;26:1275-1281.

30. Dawson SJ, Provenzano E, Caldas C. Triple negative breast cancers: clinical and prognostic implications. Eur J Cancer. 2009;45 Suppl 1: $27-40$

\section{Dovepress}

patient perspectives such as quality of life, adherence and satisfaction. The manuscript management system is completely online and includes a very quick and fair peer-review system, which is all easy to use. Visit http://www.dovepress.com/testimonials.php to read real quotes from published authors. 